\title{
Hybrid robotic coronary artery bypass grafting: how do we do it
}

\author{
Gianluca Torregrossa, Yumiko Kanei, John Puskas \\ Department of Cardiovascular Surgery, Icahn School of Medicine at Mount Sinai, NY, USA \\ Correspondence to: Gianluca Torregrossa, MD. Mount Sinai Saint Luke Hospital, 1111 Amsterdam Avenue, 10025 NYC, NY, USA. \\ Email: gtorregrossa@chpnet.org.
}

Submitted Sep 05, 2016. Accepted for publication Oct 11, 2016.

doi: 10.21037/acs.2016.11.06

View this article at: http://dx.doi.org/10.21037/acs.2016.11.06

\section{Clinical vignette}

We present the case of a 58-year-old Jehovah's Witness admitted to our Institution with unstable angina for the previous two weeks. Past medical history included hypertension, hyperlipidemia and previous testicular cancer treated with resection and chemotherapy about 15 years earlier. At admission, a trans-esophageal echocardiogram (TEE) showed preserved ejection fraction (EF) with no regional wall abnormalities or valve disease. A left heart catheterization showed two-vessel disease, including a calcified ostial left anterior descending artery (LAD) lesion and a focal right coronary artery lesion. After discussion with the patient, primary physician and interventional cardiologist, informed consent was obtained for a hybrid revascularization, including robotic coronary artery bypass grafting (CABG) with left internal mammary artery (LIMA) to LAD followed by a percutaneous coronary intervention (PCI) on the right coronary lesion with a drug eluting stent (DES).

\section{Surgical techniques}

\section{Preparation}

The patient underwent general anesthesia and was ventilated via an endotracheal tube with a bronchial blocker, isolating the left lung. A right jugular vein central line was placed, as well as an arterial line in the left radial artery. The patient was then positioned with the left side of the chest elevated approximately $30^{\circ}$ and the left elbow padded and suspended well below the posterior axillary line in order to provide adequate access to introduce the robotic instruments in the second, fifth and seventh intercostal spaces. The patient was prepped and draped in usual fashion including circumferential prepping and draping of the legs. The 'executing manipulator' of the robot (composed of three arms that will later be physically docked into the patient's left chest) is on the right side of the patient and away from the table, covered with a sterile transparent plastic drape, at this early stage. The surgical console is away from the sterile field in a corner of the operating room (OR). The scrub nurse is positioned on the left side of the patient and has both a set of robotic instruments as well as an emergency kit of surgical instruments for quick conversion to sternotomy if needed. The perfusionist, together with the cardiopulmonary bypass machine, is inside the $\mathrm{OR}$ in case they are required.

\section{Exposure}

Exposure is obtained with a camera port inserted into the left fifth intercostal space in the anterior axillary line. Two instrument ports are inserted in the second and seventh intercostal spaces in the midclavicular line under thoracoscopic vision. After docking the executing manipulator of the robot and performing robotic harvest of the LIMA, a 3-cm non-rib spreading mini-thoracotomy is performed medial to the left nipple in the fifth intercostal space for men. A 4-cm sub mammary skin incision to access the fifth intercostal space, sparing the left breast tissue, is our preference in women.

\section{Operation}

The surgery starts with single-lung ventilation of the right lung by the anaesthesiologist utilizing the bronchial blocker and insertion of the video port through a $1 \mathrm{~cm}$ skin incision in the left fifth intercostal space in the anterior axillary line by the surgeon, standing on the left side of the patient 
(Video 1). The $\mathrm{CO}_{2}$ insufflation line is connected to the video trocar port during its insertion. After $\mathrm{CO}_{2}$ insufflation at a target pressure of between 8 and $10 \mathrm{mmHg}$, further deflating the left lung, the camera is inserted and the left chest cavity inspected in order to visualize the heart within the pericardium, the diaphragm, the pulsation of the left mammary artery, the phrenic nerve and pericardio-phrenic arteries. Careful assessment of hemodynamic stability at this time is mandatory; insufflation pressures should be adjusted in order to minimize the impact of $\mathrm{CO}_{2}$ insufflation on the preload of the patient, while optimizing exposure. Thoracoscopic-assisted insertion of the two instrument ports is performed in the second and seventh intercostal spaces in the mid-clavicular line. Digital compression or insertion of a fine needle from outside the chest can help identify the correct positions for inserting the instrument ports. After this phase is completed the executing manipulator of the robot is advanced into the sterile field and the three arms are docked with the three ports inserted in the left chest. At this time, the $\mathrm{CO}_{2}$ line is switched from the camera port to the instrument port in the seventh intercostal space in order to minimize fogging condensation on the camera lens.

The surgeon now sits in the surgical console while the scrub-nurse maintains the sterile field and interchanges the instruments through the ports of the robot as needed. The camera is inserted in the camera port in the fifth intercostal space; the monopolar cautery spatula is placed in the second intercostal space port ("right hand"); and the bipolar forceps in the seventh intercostal space port ("left hand").

The LIMA is harvested as a narrow pedicle: the first cautery track is incised in the chest wall, $1 \mathrm{~cm}$ medial to the LIMA using the monopolar cautery spatula. Another cautery track is burned $1 \mathrm{~cm}$ lateral to the LIMA from near its origin at the left subclavian artery to just beyond its bifurcation at the xiphoid. Great care is taken to avoid injury to the left phrenic nerve. The harvest of the pedicle starts laterally along a broad front, working medially, gently using the bipolar forceps to retract and the monopolar cautery to burn and bluntly dissect within the correct tissue plane. The collaterals are burned carefully with the monopolar spatula. Large branches are coagulated with the bipolar cautery forceps. The bipolar forceps are used to control any residual bleeding. Large branches may also be managed with metallic clips, by exchanging either the monopolar or bipolar cautery for a clip applier instrument. The lower third of the mammary artery is generally covered by intercostal muscles that hide its course. Our tendency is to first remove the muscle in a flap from lateral to medial and then proceed with harvest of the artery under direct video visualization. When the entire length of the mammary artery has been harvested, attention is turned to the pericardium. We first create a $3-5 \mathrm{~cm}$ opening with bipolar forceps and monopolar cautery in the lateroinferior portion of the pericardium, well posterior to the course of the phrenic nerve. This opening will serve to drain any postoperative bleeding into the left pleural cavity, avoiding pericardial tamponade. A second opening of the pericardium $5-7 \mathrm{~cm}$ long, is performed anteriorly after the removal of pericardial fat; at this time we visualize the LAD artery in order to ensure the appropriate site of pericardial opening. At this point, the patient is systemically heparinised to a target ACT of 350-450 sec; this generally is accomplished with a bolus of heparin $(1.5 \mathrm{mg} / \mathrm{kg}$ or $180 \mathrm{U} / \mathrm{kg}$ ) followed by a heparin infusion at $6,000 \mathrm{U} / \mathrm{hr}$. Three white plastic clips are applied at the distal end of the LIMA, inserting them via the seventh intercostal space (left) arm of the robot. Two of the clips are deployed distally, the third one is placed $1 \mathrm{~cm}$ proximal to the previous two and has a tether consisting of a $15 \mathrm{~cm}$ piece of heavy silk suture that is later clipped to the margin of the anterior opening of the pericardium in order to facilitate retrieval of the LIMA at the time of the mini-thoracotomy. The LIMA is then divided between the clips. Careful video inspection is made throughout in order to ensure complete surgical hemostasis is achieved before removing the robotic instruments. The executing manipulator of the robot is undocked from the ports in the left side chest and removed from the sterile field. The two instrument ports in second and seventh intercostal space are then removed under direct thoracoscopic vision. Next a fine long spinal needle is inserted in the chest wall at the site where the skin incision for the non-rib spreading mini-thoracotomy is planned. This helps visualize the correct position of the skin incision relative to the anterior pericardiotomy and the site of the LAD target. After performing a $3-4 \mathrm{~cm}$ skin incision, a soft tissue retractor (Alexis Retractor ${ }^{\circledR}$, Applied Medical California) is inserted to maximize exposure. The LIMA is retrieved from the pleural space and pulled up into the incision by its tether, which was anchored by a clip at the edge of the pericardiotomy. The LIMA is prepared by injecting a solution of milrinone, blood and heparin into its distal lumen with a soft silastic bulb-tipped needle (Quest Medical, Allen, Texas). Next, an Octopus Nuvo ${ }^{\circledR}$ Tissue Stabilizer (Medtronic, Minneapolis, Minnesota) is inserted through the seventh intercostal space port site and 
advanced to the LAD target. After the head link is detached, the Octopus suction pod is passed through the minithoracotomy incision and anchored to the Nuvo device in order to stabilize the LAD. The LAD is then surrounded proximally to the site of anastomosis with a vessel loop and then opened for approximately $5-8 \mathrm{~mm}$ length in order to perform the LIMA to LAD anastomosis by off-pump coronary artery bypass (OPCAB) technique. Exquisite care is taken with this anastomosis, which must be precise in order to optimize graft patency. The humidified MisterBlower is used by a second assistant to disperse blood from the anastomotic site during suturing. After finishing the anastomosis, the LIMA pedicle is secured to the epicardium.

\section{Completion}

After completion of the LIMA to LAD anastomosis we use the Medistim MiraQ ${ }^{\mathrm{TM}}$ Cardiac transit-time Doppler device (Medistim ASA, Norway) to assess the flow in the LIMALAD graft, expecting a high flow (at least $20 \mathrm{~mL} / \mathrm{min}$ ) and a low pulsatility index score (always less than 5 and preferably less than 2.5). Finally, a chest tube is advanced through a previous port site and all surgical incisions are closed in standard fashion. Prior to closing the micro-thoracotomy, a generous intercostal block is provided with $0.5 \%$ Marcaine to improve pain control.

\section{Comments}

\section{Clinical results}

Since the arrival of the senior author of this paper (JP) at Mount Sinai Health System in 2014, we have performed 40 cases of Robotic CABG with an increase in volume and demand from our Interventional cardiologist colleagues during the last quarter of that time period. All patients had a successful operation with one conversion to sternotomy to bleeding from the IMA harvest. At our institution, the relative indications for a hybrid CABG (HCR) and PCI approach include the presence of proximal LAD disease amenable to minimally invasive LIMA-LAD bypass and the presence of non-LAD lesions considered to be amenable to PCI. Relative contraindications for HCR included a poor or poorly visualized LAD target vessel, hemodynamic instability, previous sternotomy or left thoracotomy, inability to tolerate single-lung ventilation, extreme morbid obesity, and complex, non-LAD lesions that are not suitable for PCI.

\section{Advantages}

CABG is considered the gold standard for management of patients with complex multivessel coronary artery disease. The unparalleled patency and freedom from atherosclerosis of the LIMA to the LAD graft is thought to be responsible for much of the long-term advantage of CABG over percutaneous intervention (1). Currently, PCI with drug eluting stents (DES) is appealing as it is less invasive with faster recovery and fewer short-term complications. The main drawback of PCI even in the DES era remains the high rates of re-intervention. However, current restenosis and in-stent thrombosis rates of DES are similar to the reported failure rates of SVG's, making PCI a valid alternative for revascularization of nonLAD targets (2). The aim of Hybrid CABG is to combine the strengths of CABG with those of PCI by combining the durability of the LIMA-LAD bypass graft with PCI for nonLAD lesions (3). The use of the da Vinci robot allows the surgical portion of this combined procedure to be performed with a true minimally invasive approach, facilitating a faster recovery without compromising quality of the anastomosis (4).

\section{Caveats}

Robotic CABG is technically more demanding than traditional CABG via median sternotomy. It requires a steep learning curve for both the surgeon and the OR team (cardiac anesthesia and surgical nurses) in order to become facile with the robotic techniques and instruments to perform the OPCABG portion of the LIMA-LAD anastomosis, and to be prepared to manage the challenges that can arise in case of an emergent conversion to sternotomy. Moreover, robotic CABG is feasible for patients with selected coronary anatomy; such patients represent a small niche of the entire CABG population in the current era.

\section{Acknowledgements}

None.

\section{Footnote}

Conflicts of Interest: The authors have no conflicts of interest to declare.

\section{References}

1. Harskamp RE, Vassiliades TA, Mehta RH, et al. 
Comparative Effectiveness of Hybrid Coronary Revascularization vs Coronary Artery Bypass Grafting. J Am Coll Surg 2015;221:326-34.e1.

2. Wang N, Zhou JJ, Phan S, et al. Robot-assisted Hybrid Coronary Revascularisation: Systematic Review. Heart Lung Circ 2015;24:1171-9.

3. Whellan DJ, McCarey MM, Taylor BS, et al. Trends

Cite this article as: Torregrossa G, Kanei Y, Puskas J. Hybrid robotic coronary artery bypass grafting: how do we do it. Ann Cardiothorac Surg 2016;5(6):582-585. doi: 10.21037/ acs.2016.11.06 in Robotic-Assisted Coronary Artery Bypass Grafts: A Study of The Society of Thoracic Surgeons Adult Cardiac Surgery Database, 2006 to 2012. Ann Thorac Surg 2016;102:140-6.

4. Ejiofor JI, Leacche M, Byrne JG. Robotic CABG and Hybrid Approaches: The Current Landscape. Prog Cardiovasc Dis 2015;58:356-64. 